\title{
Complexity of Social Systems
}

\author{
C. MeSJASZ* \\ Cracow University of Economics, Rakowicka 27, PL-31-510 Kraków, Poland
}

\begin{abstract}
The term "complexity" used frequently as a kind of "buzzword" has gained a specific role in the language of modern social sciences and social practice. A question is arising - how can we understand complexity of social systems/social phenomena which are characterized by limited possibility of explanation, unpredictability or low reliability of prediction? The aim of the paper is to provide a partial answer to this question. A survey of characteristics of complex systems and typology of various kinds of complexity, and of their sources is presented. It is also shown that broadly defined social systems (human systems) are affected by all kinds of complexity they are "complexities of complexities". Using the typology of interpretations of complexity as an example, it is shown what are the limitations of transferring knowledge from physics, chemistry, information theory and biology to the studies of complexity of social systems. It is especially emphasized that mathematical models, which are treated as objective when applied in social sciences must be considered as an element of intersubjective discourse.
\end{abstract}

PACS numbers: 05.45.-a, 89.65.Ef, 89.65.-s, 89.75.-k

\section{Introduction}

The term "complexity" used frequently as a kind of "buzzword" has gained a specific role in the language of modern science and social practice. At the same time "complexity scholars", i.e. the authors claiming to study complexity of nature and society, purposively or not, directly or not, stimulate expectations of policy makers by attributing marketing-like titles to their works and courses - "Hidden Order" [1], "Harnessing Complexity" [2], "Order out of Chaos" [3], "Understanding Complex Systems/Organizations" (repeated in various contexts).

Recently imagination of readers curious about the causes and consequences of the financial turmoil was mesmerized by such creatures as "Black Swan" [4] or mysterious "Dragon-Kings" running away from the power law [5], which are metaphorically reflecting the results of applications of complex systems models - the "power law" in particular, in studying that turmoil and in putting in doubt traditional statistical apparatus of theory or finance.

The demand from policy makers, military planners, bankers, financiers, managers and attempts to provide a response by the scholar community is nothing unusual by itself. A new element in the discourse between practice and "complexity studies" is resulting from awareness of limited possibility, or even impossibility of analysis and prediction of social phenomena. This impossibility is often expressed in a declaration of fuzzily defined "complexity" of the subject of research.

\footnotetext{
* e-mail: mesjaszc@uek.krakow.pl
}

A question is thus arising - how can we understand complexity of social systems/social phenomena ${ }^{\dagger}$. If limited possibility of explanation, unpredictability or low reliability of prediction is the key feature of complexity, what ideas drawn from complexity studies can help social sciences in better understanding of social systems?

This question is of a special significance in policy-oriented, normative sciences dealing with society - economics, finance, management theory, international relations, security theory, peace and conflict studies, which aim not only at description and explanation, but also at providing guidance for action.

The need or the fashion of studying complexity of society has brought about a tremendous wave of writings in which the authors, should it be sociologists, political scientists, economists, mathematicians, physicists, biologists, make attempts to describe, explain and, in particular, predict phenomena occurring in social life, and recently, especially in finance.

There are numerous writings in social sciences, economics, management and finance where authors use such concepts as system, stability, turbulence, adaptation, self-organization, equilibrium, complexity, chaos, fractal politics, attractors, catastrophes, emergence, etc. Character of applications of those concepts vary, beginning from mathematical models and ending with analogies and metaphors, with dominance of the latter two.

On the other hand, in the works rooted in broadly defined systems thinking (systems approach, systems theory), complex adaptive systems theory, complexity studies, or even more ambitiously, "complexity science", many

\footnotetext{
$\dagger$ Social system is understood herein as human system (including conscious and self-conscious actors). In general sense, social systems may also include other actors - animals or artificial agents.
} 
authors discover that their concepts used as mathematical models or as analogies and metaphors, could help to encapsulate various aspects of social reality. Such applications span from social organization at the micro-level (group, company, etc.) and end at the level of broadly defined international relations or even global system. Some of the terms associated with systems thinking and/or complexity have even become a kind of "buzzwords" of social theory and practice, frequently used in a trivialized way.

There also exists a specific "missing link" between social sciences and systems thinking, and complexity studies. On the one hand, specialists in social sciences frequently refer but to very general intuitions or simplified interpretations of system, chaos, complexity. On the other hand, scholars interested in applications of the ideas of systems and complexity to social issues ignore actual knowledge in more or less established disciplines, such as, for example, economics, management or finance. Perhaps the latter discipline constitutes an exception since studies in econophysics must be based upon competence in finance theory.

Although this exchange of ideas concerns all social sciences yet it has a special importance for normative areas - economics, political science, security studies, management and finance. In all security-oriented theories and policies, three basic human desires are expressed. First, to overcome uncertainty by enhancing predictive capabilities. Second, to set the rules of social behavior allowing to enhance the feeling of certitude, security and safety. Third, to elaborate methods to implement those rules.

Since "complexity" is the key concept in all those considerations, it is necessary to ask a question: Is it possible to describe complexity of social systems knowing that any unequivocal definition is unfeasible?

The aim of the paper is to provide at least a partial answer to this question. A survey of characteristics of complex systems and typology of various kinds of complexity and of their sources is presented. It is also shown that broadly defined social systems (human systems) are affected by all kinds of complexity - they are "complexities of complexities".

Using the typology of interpretations of complexity as an example, it is shown what are the limitations of transferring knowledge from physics, chemistry, information theory and biology to the studies of complexity of social systems. It is especially emphasized that even mathematical models, which are treated as objective when applied in social sciences must be considered as an element of intersubjective discourse.

The paper has two sources of inspiration. Firstly, it refers to development of broadly defined systems thinking/systems approach, and secondly, it is built upon the conceptual framework used by Mirowski [6-8] in his works on the mutual links between development of modern economic thought and physics, chemistry, biology and information theory.
Due to its very character - a preliminary survey and an introduction to further research, the paper does not cover the entire problem area and many issues/works have been left for further deepened considerations.

\section{Defining complex systems}

\subsection{Characteristics of complexity}

Numerous problems arise in defining terms associated with "studies of complexity", "complex systems studies", or the like. The author refrains from using the terms "complexity theory", or "complexity science" although an idea of "emerging sciences of complexity" has been already proposed by Waldrop [9]. The challenges were even reflected in the article of Horgan [10] titled: "From Complexity to Perplexity". There is not any commonly accepted definition of complexity and elaboration of such a definition seems neither needed nor achievable.

The first attempts to study complex entities go back to the works of Weaver [11] (disorganized complexity and organized complexity), Simon [12] (the architecture of complexity), and Ashby [13] (the law of requisite variety).

In his search for explaining the meaning of complexity Lloyd [14] identified 31 definitions of complexity. Later this number increased to 45 [15]. In other writings numerous definitions and interpretations of complexity and of its characteristics have been formulated and scrutinized - $[1,3,9,16-23]$.

It is obvious that a large number of definitions of complexity/complex systems leads to multitude of their characteristics. They are extensively described in the works referred to in the paper.

Assuming that no deepened discussion about definitions of systems are needed - system is an entity composed of elements and relations among them and interacting with its environment from which it is separated in a more or less fuzzy way, the following examples of characteristics and typologies of complex systems/complexity can be treated as representative ones.

The most universal characteristics of complex systems are: large number of elements and interactions, non-linearity of characteristics depicting its behavior, hierarchical structure, non-decomposability, unpredictability, self-organization.

Complexity can be also characterized by multitude of other ideas such as artificial life, autopoiesis, bifurcations, co-evolution, chaos and edge of chaos, emerging properties, far-from-equilibrium-states, fractals, instability, irreducibility, power-law, self-organized criticality, sensitivity to initial conditions ("butterfly effect"), spontaneous self-organization, which are extensively depicted in a large number of writings quoted and not quoted in this paper.

In some instances complexity studies or complexity science are identified solely with complex adaptive systems (CAS), which are treated as a specific case of multi-agent systems (MAS). There is no any commonly accepted interpretation of complex adaptive systems. Following the 
initial concepts of CAS [1], their most representative properties are as follows: non-linearity of interactions (internal and external), emerging properties and simple rules of behavior of elements, self-organization, diversity of internal structure, existence at the edge of chaos, co-evolution with environment,

The above list is obviously not complete. Complex adaptive systems are regarded at present as an instrument of modeling of collective phenomena in all disciplines of science. Due to possibility of creating elements of theoretically unlimited variety of behavior they are perceived as most promising tool of modeling of broadly defined social phenomena/social systems.

Methods applied in complexity studies include: agent-based modeling (otherwise known as generative computer simulation), cellular automata, catastrophe theory, complex adaptive systems, data mining, dynamical systems theory (otherwise known as chaos theory), fractal geometry, genetic algorithms, neural networking (otherwise known as distributed artificial intelligence), power law, scale-free networks, self-organized criticality, synergetics.

In a recently proposed typology [24], complex systems studies can be viewed as a result, or even a kind of emanation of most of the trends of broadly defined systems thinking/systems research, cybernetics and artificial intelligence. A large number of definitions and approaches to complexity puts in doubt any attempts to search for a unique meaning, or a limited number of interpretations of that concept. There are numerous domains of theory and practice where this utterance has various meanings. While it does not create problems within those domains, it may lead to distortions in communication between them.

\subsection{Types and sources of complexity}

In order to identify the meaning of complexity, based on some properties of the relationships between human observers, or observing systems in general, and all kinds of observed systems, natural and artificial, including the social ones, Biggiero ([22], p. 3, p. 6) treats predictability of behavior of an entity as the fundamental criterion for distinguishing various kinds of complexity. As a foundation he proposes an interpretation of complexity as a property of objects which are neither deterministically nor stochastically predictable. "Complexity refers to objects which are predictable only in a short run and that can be faced only with heuristic and not optimizing strategies" ([22], p. 6]).

He proposes three classes (groups) of complexity (a) objects not deterministically or stochastically predictable at all, (b) objects predictable only with infinite computational capacity, (c) objects predictable only with

\footnotetext{
¥ The term "class" is used in the paper in a non-rigorous way which is demanded in classification theory. It is rather a grouping of types.
}

a transcomputational capacity (beyond the Bremermann limit).

Coming from this typology, he defined "observed irreducible complexity (OIC)" as those states of unpredictability, which allow to classify an object in one of those three classes ([22], p. 5). Taking this typology and definition as a point of departure, it is proposed to distinguish semantically different meaning of complexity and identify its sources.

The first class of complexity, which can be called self-referential complexity, includes two classes: logical complexity and relational complexity. It relates to numerous forms of undecidability and interactions between observing systems and in some way allows one to link objective and subjective sources of complexity [25].

Logical complexity has two interrelated components. The first one, which is in some way external to the observer, can be related to Gödel's incompleteness theorem, Post's halting problem and Turing's undecidability. It can be also linked to the later works concerning the very foundations of probability theory and of mathematics in general, which have been described in the works by Kolmogorov [26] and Chaitin [27] and are used under different names - descriptive complexity, Solomonoff-Kolmogorov complexity, KolmogorovChaitin complexity, stochastic complexity, algorithmic entropy, or program-size complexity.

The sense of the "objective" component of logical complexity can be summarized that if a formal system is coherent, it is impossible to demonstrate the truth of all theorems which could be built in it. Some of them would remain undecidable, i.e. not certainly true or certainly false. It creates insurmountable limits to knowledge which can be overcome neither in infinite time nor with infinite computation power [22].

The second component of logical complexity brings about its subjective sources. In a radical constructivist approach, any single cognitive (observing) system, and specifically a human being, is also affected by self-reference due to the fact that self is also thinking itself (and about itself) $[22,25,28-30]$. Therefore undecidability affects also the observer and the very process of observation.

The second kind of self-referential complexity, derives from limitations of interactions and communication between observers, and obviously, observers/participants in social systems composed of conscious elements. It is the core of concept of "second-order cybernetics" [25] and "soft" systems thinking [31]. Leaving apart too frequently abused in social sciences the Heisenberg principle, it may be stated that interactions between observers/ participants and the system under observation as well as communication between observers/participants is never indifferent and is always exposed to distortions.

Next class of complexity and its sources, which can be called gnosiological complexity, includes two subclasses: fundamental gnosiological complexity and semiotic complexity. 
Fundamental gnosiological complexity is associated with the infinite types of distinctions that an observer can hypothetically make regarding himself/herself and in his/her own environment $[25,29,32-35]$. In this case the definition of information by Bateson ([32], p. 459) "difference which makes a difference" is the point of departure: The observer is never able to identify all differences (all information) characterizing reality (environment and himself/herself). It may be even disputable to what extent the observer in the process of construction invents or discovers reality. In the discussion going down to the biological level Maturana and Varela [28] argue that even neurophysiological signals are not objective, because the external environment reaches the brain mixed with signals and noises coming from inside of the observer.

Semiotic complexity derives from the infinite possible meanings which can be assigned by an observer to any perceived distinction/information [30, 36, 37]. It refers to the irreducible ambiguity of events, facts and information. Assigning meaning (sense-making) depends on the subjectivity (history and structure) of the observer. Subjectivity is also strongly dependent on language and culture. The same fact can be interpreted differently by different actors. It not only affects the understanding but also influences the communication, as it was already observed for relational complexity.

The third class of complexity is derived from the infinite developments a nonlinear system can perform. It can be called chaotic and non-equilibrium complexity and was initially assigned by Biggiero [22] to the same class as fundamental gnosiological and semiotic complexity. It seems, however, that due to its specificity this type of complexity should be considered as a separate type yet linked to the second class of complexity.

This type of complexity covers a vast area of nonlinear dynamic systems including such ideas as singularity theory, bifurcation theory, catastrophe theory and fractals geometry.

Chaos emerges when a dynamic system is highly sensitive to initial conditions or when it has numerous bifurcations or singularities. In consequence, dynamic systems become unpredictable since many different trajectories may be followed and many different fixed points (attractors) may emerge.

According to [38] and [22], chaos, as well as dissipative structures [3] and complex adaptive systems (CAS) $[1,9,17]$, share a common property of ability of self-organizing. Self-organizational complexity is associated with unexpected appearance of ordered systems from a disordered aggregation of elements, e.g. self-organized criticality [18], synergetics [21]. Although common in physics and chemistry, self-organization is regarded as a specific feature of biological phenomena [17] and social phenomena [30, 38-40]. Various applications of power law $[4,5,41]$ as well as scale-free networks [23] can also be added to this class of complexity and its sources.

The fourth class of complexity is called computational complexity. It can be identified with logical intractability and computational intractability. Logical intractability to a large extent overlaps with logical complexity, especially in the concepts developed in the second half of the 20th century by Solomonoff, Kolmogoroff [26] and Chaitin [27].

Computational complexity occurs when it is impossible to end computing either because of infinite or "practically excessive" resources needed for computation (time or computing power). Computational (algorithmic) complexity theory concerns intractability in measuring computational resources in terms of program execution time.

In simplest way it can be described as follows. Depending on the type of solvability by different Turing machines the polynomial time algorithms, called NP class, can be divided into three classes. First, class $\mathrm{P}$ concerns algorithms solvable with a deterministic Turing machine. Second, class NP-complete, concerns algorithms solvable only with a nondeterministic Turing machine. Third, class NP-intermediate, also deals with algorithms solvable only with a nondeterministic Turing machine.

Both NP problems solvable only with a nondeterministic Turing machine are considered as intractable with an exception of small inputs. Problems belonging to class $\mathrm{P}$ are treated as tractable, but with a very high limits to input size. Due to the height of the limits they are regarded as already intractable [42]. Computational complexity is a subject of intensive studies and the above explanation is an introduction to more advanced considerations [43].

The above typology can be summarized with an approach taken from information theory. According to the rudimentary interpretation, information can be described with quantitative (syntactical) and qualitative (semantic) aspects [44]. This division is equivalent to the pairs: objective vs. subjective, or independent of observer and observer-dependent, and independent of meaning and meaning dependent. Computational, chaotic ad logical complexity and its sources may be assigned to the first class while gnosiological, semiotic and relational complexity and its sources are associated with meaning and natural languages.

Two conclusions important in studying any complex systems, and social systems in particular can be drawn. Firstly, in all discussions on complexity of social systems composed of conscious elements the role of observer-participant must be always taken into account, even when studies concern objectively defined complexity. This postulate does not necessary mean acceptance of radical constructivism in which observer invents reality. It only should be remembered when quantitative modeling of social systems is conducted that any of the models is absolutely objective.

Second, human systems are characterized by the presence of all sources and types of complexity [22]. It may be then summarized that in a universal sense all many collective phenomena may be complex, including for example animal or artificial social systems but human systems made of conscious elements are the "complexities of complexities". 


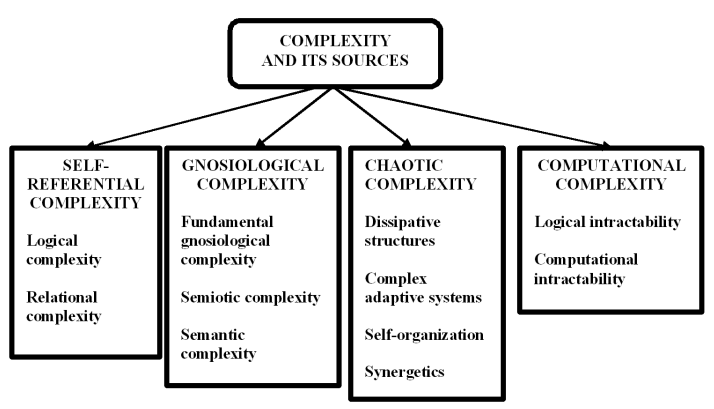

Fig. 1. Types of complexity and its sources. Source: own research and after [22].

\section{3. "Hard" and "soft" complexity}

The above typologies can be supplemented with another division relevant to social sciences methodology. This division is also a summary of different streams of systems thinking/complex systems studies. The first stream developed only with the use of mathematical modeling can be called "hard" complexity research as an analogy with "hard" systems thinking ${ }^{\S}$. The "soft" complexity research, also coined per analogy with "soft" systems thinking [31], includes qualitative concepts of complexity elaborated in other areas - cybernetics and systems thinking, social sciences and in psychology. It is necessary to stress that soft complexity initially had two domains - purely verbal considerations on complexity, and applications of the ideas from "hard" complexity in qualitative considerations. At present, under the impact of "hard" complexity only one stream of soft complexity studies exists [24].

Subjectivity/qualitative methods are the main aspect of complexity in the "soft" approach. It is just a consequence of the fact that complexity is not an intrinsic property of an object but rather depends on the observer.

In social sciences, and particularly in sociology, special attention is given to the concepts of complexity of social systems proposed by Luhmann. First of all, as one of a few authors, he made an attempt to elaborate a comprehensive definition of a social system based solely on communication and on the concept of autopoiesis (self-creation) of biological systems. Autopoiesis means "auto (self)-creation" (from the Greek: auto - $\alpha v \tau o$ for self-

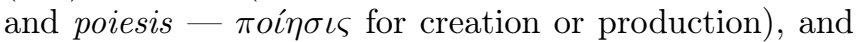
expresses a fundamental dialectic between structure and function. The concept of autopoiesis was introduced by Chilean biologists Maturana and Varela in the early 1970 s. It was originally presented as a system description that was designed to define and explain the nature of living systems.

According to its authors, "An autopoietic machine is a machine organized (defined as a unity) as a network

$\S$ The term soft complexity science was also used in [45]. of processes of production (transformation and destruction) of components which: (i) through their interactions and transformations continuously regenerate and realize the network of processes (relations) that produced them; and (ii) constitute it (the machine) as a concrete unity in space in which they (the components) exist by specifying the topological domain of its realization as such a network" ([28], p. 78).

The space defined by an autopoietic system is self-contained and cannot be described by using dimensions that define another space. When we refer to our interactions with a concrete autopoietic system, however, we project this system on the space of our manipulations and make a description of this projection ([28], p. 89).

The most representative example of an autopoietic system is the biological cell, which being based on an external flow of molecules and energy, produce the components which, in turn, continue to maintain the organized bounded structure that gives rise to these components. An autopoietic system is autonomous and operationally closed, in the sense that there are sufficient processes within it to maintain the whole.

An autopoietic system stands in opposition to an allopoietic system (externally created). Allopoietic is the process whereby an organization produces something other than the organization itself, e.g. an assembly line.

It is worthwhile to mention that Maturana and Varela drew inspiration from the concept of distinction developed by Spencer-Brown [46].

Autopoiesis refers also to the self-reference and the role of observer. It is reflected in the assertion: "everything said is said by an observer" ([28], p. xix). Due to such a self-referential approach the concept of autopoiesis was criticized as a form of solipsistic methodology and radical constructivism.

The concept of autopoiesis was used by Luhmann to elaborate an indigenous theory of social systems, which has become one of most popular universal social theories. He defines social system of conscious units as autopoietic system of meaningful communication. Autopoiesis refers not to the tangible attributes of systems but to communication $[30,40]$.

In a preliminary and simplified interpretation of intricate Luhmann's ideas, social systems operate at the level of a re-entry of their form into their form - concept taken directly from biological autopoiesis [28] and indirectly from [46]. It also means that description of the system is a part of the system. Since they use their own output as input they cannot compute their own states but they are "autopoietic" systems, and that means that they are their own product. In this interpretation, social system are thus self-reflexive and self-referential.

The theories of social systems proposed by Luhmann are broadly discussed in social theory, especially in Europe. When recalling Luhmann, it is necessary to realize the controversy about the laws of form elaborated by Spencer Brown [46] and to know that even the co-founder of biological autopoiesis, Maturana criticized the use by 
Luhmann of that idea in studying social systems ([47], p. 78).

The Luhmann concept of "soft" complexity is likely its most influential interpretations in contemporary social theory. According to this concept, a complex system is one in which there are more possibilities than can be actualized. Complexity of operations means that the number of possible relations becomes too large with respect to the capacity of elements to establish relations. It means that complexity enforces selection. The other concept of complexity is defined as a problem of observation. Now, if a system has to select its relations itself, it is difficult to foresee what relations it will select, for even if a particular selection is known, it is not possible to deduce which selections would be made ([30], p. 81).

The idea of complexity of Luhmann is also applied in defining risk in social systems. The large amount of elements in a given system means that not all elements can relate to all other elements. Complexity means the need for selectivity, and the need for selectivity means contingency, and contingency means risk [48].

Complexity of social system developed by Luhmann is strongly linked to self-reference since reduction of complexity is also a property of the system's own self-observation, because no system can possess total self-insight. This phenomenon is representative for epistemology of modern social sciences, where observation and self-observation, reflexivity and self-reflexivity, and subsequently, self-reference an recursion are playing a growing role. According to this interpretation, social systems are becoming self-observing, self-reflexive entities trying to solve arising problems through the processes of adaptation (learning).

A deeper look into subjective aspects of complexity leads to the conclusion that any cognitive system must include at least a partial projection of its environment and of itself onto itself. A definition of complexity referring to that idea was proposed by Rosen, who also elaborated the concept of anticipatory system, i.e. a system containing a predictive model of itself and/or its environment, which allows it to change state at an instant in accord with the model's predictions pertaining to a latter instant ([49], p. 341). According to Rosen, a system is simple if all its models are simulable which is approximately equivalent to Turing-computability. A system that is not simple, and that accordingly must have a nonsimulable model, is complex ([50], p. 392).

The concepts of Rosen's anticipatory systems, has been supplemented by the ideas of incursion (inclusive or implicit recursion) and hyperincursion (incursion with multiple solutions) developed by Dubois [51].

Using the concept of Luhmann's complexity, Qvortrup has put before the concept of hypercomplexity. He linked Simon's "bounded rationality" as a limitation to choice (selection) with the complexity resulting from impossibility to make that selection. Hypercomplexity is complexity inscribed in complexity, e.g. second-order complexity. As an example, hypercomplexity is the result of one ob- server's description of another observer's descriptions of complexity, or it is the result of a complex observer's description of its own complexity [52].

\section{Complexity and social systems:}

\section{Mathematical models, analogies and metaphors}

\subsection{Reflexivity and self-reflexivity of social systems}

Application of the concept of complexity with its multitude of interpretations in social sciences is becoming even more difficult due to another similar obstacle multitude of meanings of social systems. Even if the hierarchy of social systems viewed from a global level to a group scale is left apart, it is not possible to comprehend all meanings of social systems. The basic assumption is that social systems are mental constructs of the observers (participants) as interpretations of behavior of their components and of the entities.

The most important distinction in defining social systems lies thus in the role of participant-observer. If she/he remains outside the system and is not able to interfere into its behavior, then a physicalist approach can be applied, obviously without possibility to refer to quantum mechanics. Such an approach belongs to the tradition of the "first order cybernetics" or the "hard" systems thinking. If the participant/observer is able to exert an impact on the system, then consequences of reflexivity and self-reflexivity must be taken into account. Under such circumstances the "second order cybernetics" or the "soft" systems thinking are the basic methods of research $[25,31]$.

Complexity of social systems is more difficult to comprehend, since it is always a result of an intersubjective discourse. The "hard" approach allows us for more precisely defined tangible attributes of the system described by measurable with "strong" ratio scale tangible characteristics. The "soft" approach makes the description much difficult since intersubjectivity depends on transfer of imprecise meanings in the discourse. In both cases it is necessary to consider limitations stemming from reification of subjective/intersubjective categories. It may thus be concluded that if studies concentrate upon "tangible", measurable attributes of social systems, then "hard" complexity methods, mainly mathematical models, including simulations, can be applied. Otherwise, the discussion must also include reflexive ideas taken from "soft" complexity studies. Therefore a mixed approach is necessary - mathematical modeling and/or analogies and metaphors .

There is a specific factor allowing to distinguish traditionally defined systems thinking from complexity research, at least until the mid-1980s. While systems thinking sought for holistic ideas and universal patterns in all

I The terms analogies and metaphors are applied in the paper in a simplified sense. There are also other linguistic concepts, which should be applied in more advanced considerations on modeling and problem solving [53]. 
kinds of systems, complexity research defined its goals in a more specific manner. A common theoretical framework, the vision of underlying unity illuminating nature and humankind is viewed as an epistemological foundation of complexity studies [9]. This claim for unity results from an assumption, that there are simple sets of mathematical rules that when followed by a computer give rise to extremely complicated, or rather complex, patterns. The world also contains many extremely complicated patterns. Thus, in consequence it can be concluded that simple rules underlie many extremely complicated phenomena in the world. With the help of powerful computers, scientists can root those rules out. Subsequently, at least some rules of complex systems could be unveiled.

Although such an approach was criticized, as based on a seductive syllogism $[10,45]$, it appears that it still exists explicitly or implicitly in numerous works in the hard complexity research.

There exists another, often omitted, barrier in defining tangible attributes of social systems. In some instances measurable characteristics are easily identifiable, e.g. physical aspects or financial measures expressed directly in monetary units. In some cases an intangible category is reified as to make it measurable. The discussion on "knowledge management" or on "knowledge based economy" provides best examples. Operationalization of the concept of knowledge not only in economics but also in other social sciences is usually based upon a twofold approach. On the one hand, several "tangible" attributes of social systems are treated as more or less representative for the state of knowledge, e.g. number of patents, income for innovation. On the other, attempts are made to make some unquantifiable characteristics measurable at least with the cardinal scale in order to use them in rating and scoring methods [54].

Operationalization of intangible attributes of social system always creates a trap of reification. In consequence, even mathematically elegant models may have a limited validity since they result from false or at least dubious assumptions.

\subsection{Linguistic approach to complexity of social systems}

All the above barriers of interpretations of social systems complexity can be also explained with a linguistic approach. Systems thinking/complex systems studies, or whatever it may be called, can be used in social sciences as sources of analogies, metaphors and mathematical models. According to this distinction, the term "(formal) models" is used only for mathematical structures.

Using another approach, our questions refer to three Wittgenstein's [35] "language games" including meaning of three utterances: (1) the meaning of social systems, (2) the meaning of complexity and (3) the meaning of ideas in which the concepts of social systems and the concept of complexity are together applied.

Mathematical models can be applied in three areas of complexity studies: computing-based experimental mathematics, high precision measurement made across various disciplines and confirming "universality" of complexity properties, and mathematical studies embodying new analytical models, theorems and results.

Models, analogies, and metaphors deriving from systems thinking and complexity studies are gaining a special significance in social sciences. For mathematical models it is quite obvious that they are associated with "objective" research. Analogies and metaphors taken from complex systems studies are related to ideas drawn from "rational" science. They are treated as "scientific" and may provide additional political influence in the discourse resulting from "sound" normative (precisely prescriptive), legitimacy in any policy-oriented debate.

In applications of models, analogies and metaphors in social sciences the following approaches can be identified: descriptive, explanatory, predictive, anticipatory, normative, prescriptive, retrospective, retrodictive (backcasting), control and regulation.

Bell et al. [55] have proposed to discern between the normative approach resulting from mathematical models, predominantly game models, and prescriptive approach reflecting recommendations resulting from decision analysis, including also qualitative aspects.

Following the distinction from traditional cybernetics, control and regulation approach can be also proposed. In management this approach is expressed in a way the dominant analogy or metaphor influences control of a system, i.e. they differ for mechanistic, evolutionary or learning system $[56,57]$. Limitations of prediction of behavior, design and control of complex systems impose also other ways of managing complex systems. Axelrod and Cohen ([2], p. xvi) proposed to "harness" complexity of social systems: "... to convey a perspective that is not explanatory but active - seeking to improve but without being able fully to control".

Complexity associated with non-linear dynamics adds some new elements to our knowledge of social dynamics. We do not only become aware that social systems are uncontrollable, but even desirability of such control is already put in doubt. Self-organization is regarded as a desired pattern of dynamics in economics and politics. It was already reflected in the interests of Hayek [58] in complexity of social systems as an argument against centrally planned economy.

Another lesson of non-linear dynamics and complex systems teaches us is that social changes, or in a broader sense, evolution, are produced by both deterministic historical factors and chance events that may push social phenomena to new patterns of behavior. Thanks to better understanding the confluence of chance and determinism in social systems we may better learn what kind of actions we have to undertake, or even perhaps, what kind of norms we have to apply.

It must be also reminded that analogies and metaphors of rather loosely interpreted non-linearity, chaos, complexity, self-organization, etc. in many instances have become the backbone of the post-modernist (post- 
-structuralist) new science. Reaffirmation of limited predictability has become an epistemological foundation of the discourse-based science. Numerous examples can be quoted but as an illustration it is worthwhile to recall synthesis of post-modernist ideas of Braudel and Priogogine's concepts on far-from-equilibrium states made by Wallerstein ([59], p. 160-169) in modeling social systems, although solely at a metaphorical level.

The above epistemological links between complexity research and social sciences are predominantly associated with the "hard" complexity. The input to this area exerted by the "soft" complexity research is equally significant. Reflexive complexity of society has become one of the foundations of post-modern social theory [30].

Unfortunately, various abuses and misuses may occur, when analogies and metaphors drawn from the "hard" complexity research, and to a lesser extent from the "soft" complexity research are treated too carelessly even by eminent social theoreticians of post-modernism/post-structuralism. Several examples of such abuses are mirrored in the so-called "Sokal Hoax" and other examples widely described by the originator of that hoax [60].

Summarizing the above considerations, it may be concluded that applications of complex systems analogies and metaphors in social sciences expose two basic weaknesses. First, in most of their applications it has been omitted that they can be predominantly used solely as descriptive and explanative instruments. Application of those analogies and metaphors for prediction and norms-setting is always limited by their reification. It brought about double consequences. In theoretical research many futile efforts were made to make them more "scientific", "objective" and "analytical". Practice, in turn, has been enriched with "objective" terms with various hidden normative loading, e.g. "stability", "equilibrium". Some of these problems could be avoided if the following phenomenon were taken into account. It can be ironically called "The Law of Metaphorical Infinity of Social Systems":

Any theory and/or model elaborated in physics, chemistry, biology, automatic control theory, etc., in order to study collective phenomena ("systems thinking", "complexity studies"), can be applied as a source of analogies and metaphors in various attempts aiming at description, explanation (sometimes even prediction and prescription), of phenomena taking place in social systems, beginning from small groups, and ending with the world system.

The Law can be supplemented with an observation that large-scale social systems seem particularly tempting for such efforts. It is easy to compare a society to a machine or biological system. It will be much more difficult to do the same with a small group or family. The meaning of the term "society" is much broader and more distant from our everyday experiences. In the case of a smaller group we can easier identify the humans as elements of the system.
Second, along with the differentiation, pluralism and multipolarity associated with the democratisation and expansion of the market economy along with their limited effectiveness, e.g. the recent turmoil on financial markets, more attention is given to concepts drawn from complex systems studies. However, stress must be put not only on the "objective" soundness and mathematical elegance of these concepts. Instead, they should be seen as analogies and metaphors reflecting observer-related, emotionally-laden and normative understandings of the world, which not always can be operationalized. Thus in studies which intend to deal with the old and new systems thinking-related terms: autopoiesis, chaos, complexity, equilibrium, fluctuation, homeostasis, non-equilibrium, self-organisation, stability, synergy, turbulence, ultrastability, etc., it is necessary to concentrate on the semantic foundations before any applications and operationalizations in studies of social systems.

It must be also remembered that contrary to physics, mathematical modelling in social sciences, including obviously complex systems, is rather rarely based upon axiomatic approach, perhaps with an exception of mathematical (theoretical) economics. In other cases an approach based upon operationalization is applied. But in such case the process of building operationalizable definitions begins from "central metaphor" or "stylized fact", a qualitative idea which is later developed with the use of formal models, e.g. distribution, risk [53]. In such case the selection of the model is subjective in all possible ways - self-reflexive and self referential (from the point of view of the modeller).

As the final example of subjectivity of mathematical models applied in studying social systems a dispute between authors of models applied in finances can be recalled.

The divide in the dispute between forecasting in finances based on classical econometric models, and models based on broadly defined complexity methods, gained additional momentum in 2007-2008. The divide was made popular by the already mentioned "Black Swan" [4] although other more or less known works, e.g. [61] dealt with those issues well before the crisis.

The most extreme position in that dispute has been recently taken by Farmer and Foley [62], who exposed validity of agent-based modeling (complex adaptive systems) in finance as reflecting nonlinearity and non-equilibrium states, although at the same time admitting that those models are still at a preliminary stage of development.

They add that there is no any sufficiently developed analytical methods in financial policy at the state level. Two quotations seem representative for their position. "This is hard for most non-economists to believe. Aren't people on Wall Street using fancy mathematical models? Yes, but for a completely different purpose: modeling the potential profit and risk of individual trades. There is no attempt to assemble the pieces and understand the behavior of the whole economic system" ([62], p. 685). "The 
policy predictions of the models that are in use aren't wrong, they are simply non-existent" ([62], p. 686).

\section{Conclusions}

The paper should be treated as a survey aiming at systematization of areas of research and as support in avoiding basic misunderstandings in the discussion on complexity of broadly defined social systems. The main conclusion stemming from the paper is that all characteristics assigned to complex systems are relevant to social systems composed of conscious actors. This rather evident observation leads to other conclusions. The multi-faceted complexity of social systems is always deriving from an intersubjective character of those systems. Complexity studies show that in all research of social systems the role of an observer/participant must be always taken into account, directly or indirectly.

In addition, if any aspect of complexity of a social system is under scrutiny, it should be precisely conceptualized in quantitative terms as to be subsequently applied for building a mathematical model.

Finally, awareness of inherent intricacy of social systems should help in deeper understanding of the limits of applying formal models for prediction of their behavior.

The links between "hard" and "soft" complexity of social systems are likely the most promising direction of future interdisciplinary studies.

\section{References}

[1] J.D. Holland, Hidden Order: How Adaptation Builds Complexity, Basic Books, New York 1995.

[2] R. Axelrod, M.D. Cohen, Harnessing Complexity: Organizational Implications of a Scientific Frontier, The Free Press, New York 1999.

[3] G. Nicolis, I. Prigogine, Exploring Complexity, W.H. Freeman and Company, New York 1989.

[4] N.N. Taleb, The Black Swan: The Impact of the Highly Improbable, Random House, New York 2007.

[5] D. Sornette, Int. J. Terraspace Sci. Eng. 2, 1 (2009).

[6] P. Mirowski, More Heat than Light: Economics as Social Physics, Physics as Nature's Economics, Cambridge University Press, Cambridge 1989.

[7] Natural Images in Economic Thought: "Markets Read in Tooth $\&$ Claw", Ed. P. Mirowski, Cambridge University Press, Cambridge 1994.

[8] P. Mirowski, Machine Dream: Economics Becomes a Cyborg Science, Cambridge University Press, Cambridge 2002 .

[9] M.M. Waldrop, Complexity: The Emerging Science at the Edge of Order and Chaos, Simon \& Schuster, New York 1992

[10] J. Horgan, Scientific American 272, 104 (1995).

[11] W. Weaver, American Scientist 36, 536 (1948).

[12] H.A. Simon, Proc. Am. Philos. Soc. 106, 467 (1962).

[13] W.R. Ashby, An Introduction to Cybernetics, Chapman and Hall, London 1956.
[14] S. Lloyd, in: 1989 Lectures in Complex Systems, Ed. E. Jen, Addison-Wesley, Redwood City (CA) 1990, p. 67 .

[15] S. Lloyd, IEEE Control Syst. Mag. 21, 7 (2001).

[16] M. Gell-Mann, Complexity 1, 16 (1995).

[17] S.A. Kauffman, At Home in the Universe: The Search for Laws of Self-Organization and Complexity, Oxford University Press, New York 1995.

[18] P. Bak, How Nature Works: The Science of Self-Organized Criticality, Copernicus/Springer, New York 1996

[19] Y. Bar-Yam, Dynamics of Complex Systems, Addison-Wesley, Reading (MA) 1997.

[20] J. Gleick, Chaos: The Making of a New Science, Viking Press, New York 1987.

[21] H. Haken, Discrete Dynamics in Nature and Society 1, 1 (1997).

[22] L. Biggiero, Nonlinear Dynam. Psychology Life Sci. $\mathbf{5}, 3(2001)$.

[23] A.L. Barabási, Linked. How Everything is Connected to Everything Else and What It Means for Business, Science, and Everyday Life, Penguin, New York 2003.

[24] B. Castellani, F.W. Hafferty, Sociology and Complexity Science: A New Field of Inquiry, Springer, Berlin 2009.

[25] H. von Foerster, Observing Systems, Intersystems Publications, Seaside (CA) 1982.

[26] M. Li, P. Vitányi, An Introduction to Kolmogorov Complexity and Its Applications, Springer, New York 2008.

[27] G.J. Chaitin, Exploring Randomness, Springer, London 2001.

[28] H. Maturana, F. Varela, Autopoiesis and Cognition: The Realization of the Living, D. Reidel Publishing Co., Dordrecht 1980.

[29] The Invented Reality: How Do We Know What We Believe We Know? (Contributions to Constructivism), Ed. P. Watzlawick, W.W. Norton and Co. New York 1984.

[30] N. Luhmann, Essays on Self-Reference, Columbia University Press, New York 1990.

[31] P.B. Checkland, Systems Thinking, Systems Practice, Wiley, New York 1998.

[32] G. Bateson, Steps to an Ecology of Mind: Collected Essays in Anthropology, Psychiatry, Evolution, and Epistemology, Chandler, San Francisco 1972.

[33] G. Bateson, Mind and Nature: A Necessary Unity, Bantam Books, New York 1979.

[34] L. Wittgenstein, On Certainty, Blackwell, Oxford 1969.

[35] L. Wittgenstein, Philosophical Investigations. the german text with a revised English translation, 3rd ed., Blackwell, Oxford 2002.

[36] C.S. Peirce, Collected Papers, Belknap Press, Cambridge (MA) 1931

[37] U. Eco, A Theory of Semiotics, University of Indiana Press, Bloomington 1979.

[38] J.L. Casti, Complexification: Explaining a Paradoxical World Through the Science of Surprise, HarperCollins, New York 1994. 
[39] L. Leydesdorff, A Sociological Theory of Communication: The Self-Organization of the Knowledge-Based Society, Universal Publishers/uPUBLISHCOM, USA 2001.

[40] N. Luhmann, Die Gesellschaft der Gesellschaft, Suhrkamp, Frankfurt am Main 1997.

[41] B. Mandelbrot, Fractals and Chaos: The Mandelbrot Set and Beyond, Yale University Press, New Haven 2004.

[42] M.R. Garey, D.S. Johnson, Computers and Intractability. A Guide to the Theory of NP-Completeness, W.H. Freeman and Co., New York 1979.

[43] S. Arora, B. Barak, Computational Complexity. A Modern Approach, Cambridge University Press, New York 2009.

[44] L. Brillouin, Science and Information Theory, Academic Press, New York 1956.

[45] K. Richardson, P. Cilliers, Emergence 3, 5 (2001).

[46] G. Spencer, Brown, Laws of Form, The Julian Press, New York 1972

[47] B. Poerksen, The Certainty of Uncertainty. Dialogues Introducing Contructivism, Imprint Academic, Exeter 2004.

[48] N. Luhmann, Risk: A Sociological Theory, Aldine de Gruyter, New York 1993.

[49] R. Rosen, Anticipatory Systems: Philosophical, Mathematical and Rosen Methodological Foundations, Pergamon Press, New York 1985.

[50] R. Rosen, Essays on Life Itself, Columbia University Press, New York 1998.
[51] D.M. Dubois, AIP Proc. 437, 3 (1998).

[52] L. Qvortrup, The Hypercomplex Society, Peter Lang, New York 2003.

[53] G. Lakoff, in: Metaphor and Thought, Ed. A. Ortony, Cambridge University Press, Cambridge 202 (1993).

[54] B. Lev, Intangibles. Management, Measurement and Reporting, Brooking Institution Press, Washington, DC, 2001.

[55] D.E. Bell, H. Raiffa, A. Tversky, Decision Making: Descriptive, Normative, and Prescriptive Interactions, Cambridge University Press, Cambridge 1988.

[56] P.M. Senge, The Fifth Discipline: The Art and Practice of the Learning Organization, Doubleday, New York 1990.

[57] I. Palmer, R. Dunford, Acad. Managem. Rev. 21, 691 (1996).

[58] F.A. Hayek, in: Studies in Philosophy, Politics, and Economics, Ed. F.A. Hayek, Routledge and Kegan Paul, London 1967, p. 22.

[59] I. Wallerstein, The Essential Wallerstein, The New Press, New York 2000.

[60] A. Sokal, J. Bricmont, Fashionable Nonsense. Postmodern Intellectuals' Abuse of Science, Picador, New York 1998.

[61] B. Mandelbrot, R.L. Hudson, The (mis)Behavior of Markets. A Fractal View of Risk, Ruin, and Reward, Basic Books, New York 2004.

[62] J.D. Farmer, D. Foley, Nature 460, 685 (2009). 